\title{
¿Está Centroamérica y el Caribe preparada para las ciudades del futuro?
}

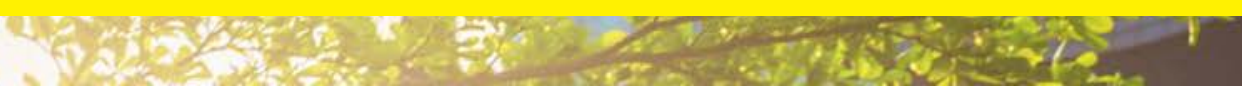

Por Ernest \& Young El Salvador S.A. de C.V.

\section{Tendencias de las ciudades del mundo}

Las ciudades son asentamientos de población con atribuciones y funciones político-administrativas, económicas y religiosas. Esto tiene un reflejo material en la presencia de edificios específicos y en su configuración urbanística. Los cuatro retos claves que enfrentan las ciudades de todo el mundo son:

\section{Población:}

\section{$70 \%$}

De la población vivirá en zonas urbanas para el 2050.
41

Ciudades para 2030 serán mega ciudades con más de 20 millones de personas.

\section{$50 \%$}

De la población vive en ciudades actualmente.
500 Ciudades

En un plazo no mayor a 10 años tendrán más de un millón de personas.

\section{Seguridad:}

\section{0 millones \\ $26-45 \%$}

De personas viven en países afectados por ciclos repetidos de violencia criminal y política.
Aumento estimado de los disturbios civiles

(Protestas, violencia por

causas políticas y sociales).

\section{$\$ 400$ billones}

De la población vive en ciudades actualmente.

\section{Infraestructura:}

\section{\$94 Billones}

Se necesitan para inversiones globales en infraestructura para el 2040.

\section{$1 / 5$}

De este dinero podría quedar sin financiamiento si continúan las tendencias actuales de gasto.

\section{7 billones}

Se necesitan cada año para inversiones de infraestructura para cubrir la demanda.

\section{\$1 trillón}

es el costo estimado de la contaminación atmosférica en los países de la OCDE debido a las emisiones del transporte por carretera.

\section{$90 \%$}

de las áreas urbanas del mundo son costas que enfrentan el peligro del aumento en el nivel del mar.

\section{+1.1 billón}

Número de personas mayores de 60 años que habrá para el 2050, lo que resulta en un aumento de los costos de salud y la carga financiera.

Las ciudades pronto representarán el $\mathbf{9 0} \%$ del crecimiento de la población mundial, el $\mathbf{8 0} \%$ de sus emisiones de carbono y el $75 \%$ del consumo de energía. La pregunta que surge es: ¿Qué estamos haciendo al respecto? 


\section{Ciudades del Futuro en la Región.}

Lo expuesto anteriormente nos permite entender el grado de urgencia a la hora de desarrollar ciudades preparadas para el futuro, a nivel global cientos de iniciativas prevén el cómo las ciudades pueden responder de la mejor forma ante estos retos.

Sin embargo regionalmente, aún cuando el tema es una tendencia informativa, la ejecución del mismo se encuentra distante, ya que culturalmente para Centroamérica y el Caribe las ciudades inteligentes son vistas como un tema futurista o bien el tema se reduce únicamente al uso de la tecnología.

\section{Es por esto que EY ha desarrollado el presente estudio para mostrar el estatus regional de nuestras capitales y promover las ciudades del futuro como una realidad para nuestros países.}

Empecemos por entender las dos características más importantes que deben tener las ciudades del futuro:

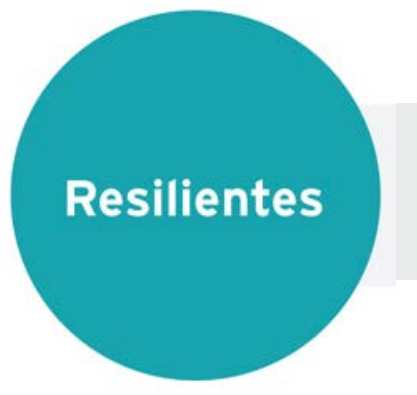

1. La resiliencia urbana es la capacidad de los individuos, comunidades, instituciones, empresas y sistemas dentro de una ciudad para sobrevivir, adaptarse y prosperar, sin importar las tensiones crónicas y problemas complejos que enfrenten.

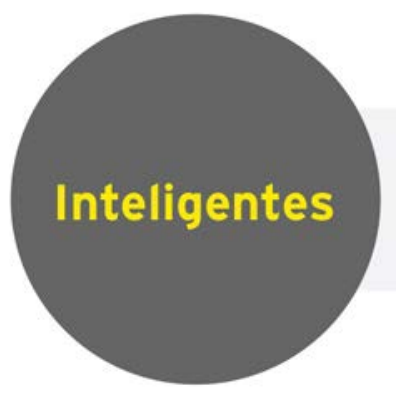

2. Una ciudad inteligente es aquella que hace uso de los avances tecnológicos para mejorar la calidad de vida de sus habitantes, promueve el desarrollo sostenible, optimiza los recursos disponibles y brinda una participación ciudadana activa.

A través de estos dos conceptos es posible entender que las ciudades que necesitamos no son espacios con vehículos voladores, hologramas, cien por ciento tecnológicas y libres de cualquier problema, por el contrario son espacios donde es posible sobreponerse a las dificultades de formas innovadoras, en uno o varios temas de forma paulatina.

Teniendo claros estos conceptos, podemos abordar los números que definen el estado general de nuestras ciudades en las siguientes áreas:
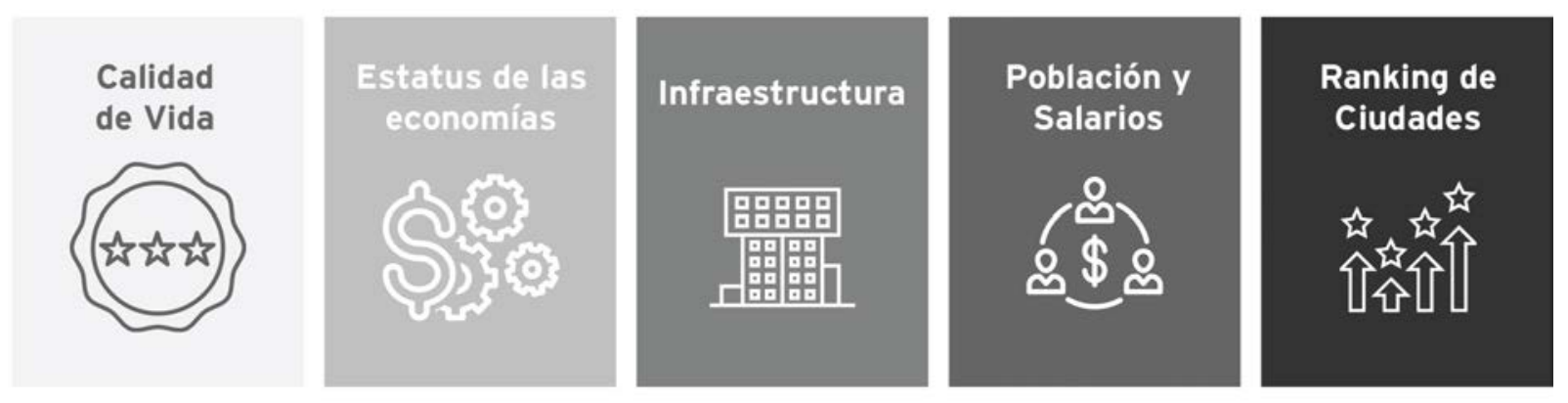

Fuente: Estudio de Ciudades Inteligentes EY Italia 2017. 


\section{Estado General de las Ciudades}

\section{Calidad de vida}

Calidad de vida es un concepto que hace alusión a varios niveles de generalización pasando por sociedad, comunidad, hasta el aspecto físico y mental. Es decir, las condiciones en que vive un ser humano y que determinan una vida digna y placentera. Además está influido por la cultura en la que vive, los valores y la sociedad.

El estudio de Mercer es uno de los más detallados del mundo sobre calidad de vida, el ranking de este año incluye 231 de esas ciudades.

A continuación el extracto del ranking regional:

\begin{tabular}{|c|}
\hline Calidad de vida \\
\hline Ránking América Latina \\
\hline 79. Montevideo (Uruguay) \\
\hline 93. Buenos Aires (Argentina) \\
\hline 95. Santiago (Chile) \\
\hline 97. Ciudad de Panamá \\
\hline 109. Brasilia (Brasil) \\
\hline 110. Monterrey (México) \\
\hline 110. San José (Costa Rica) \\
\hline 115. Asunción (Paraguay) \\
\hline 118. Río de Janeiro (Brasil) \\
\hline 121. Quito (Ecuador) \\
\hline 121. Sao Paulo (Brasil) \\
\hline 124. Lima (Perú) \\
\hline 127. Manaos (Brasil) \\
\hline 128. Ciudad de México \\
\hline 129. Bogotá (Colombia) \\
\hline 139. Santo Domingo (República Dominicana) \\
\hline 147. Puerto de España (Trinidad y Tobago) \\
\hline 155. Ciudad de Guatemala (Guatemala) \\
\hline 157. La Paz (Bolivia) \\
\hline 174. Managua (Nicaragua) \\
\hline 183. El Salvador (EI Salvador) \\
\hline 188. Tegucigalpa (Honduras) \\
\hline 189. Caracas (Venezuela) \\
\hline 192. La Habana (Cuba) \\
\hline
\end{tabular}

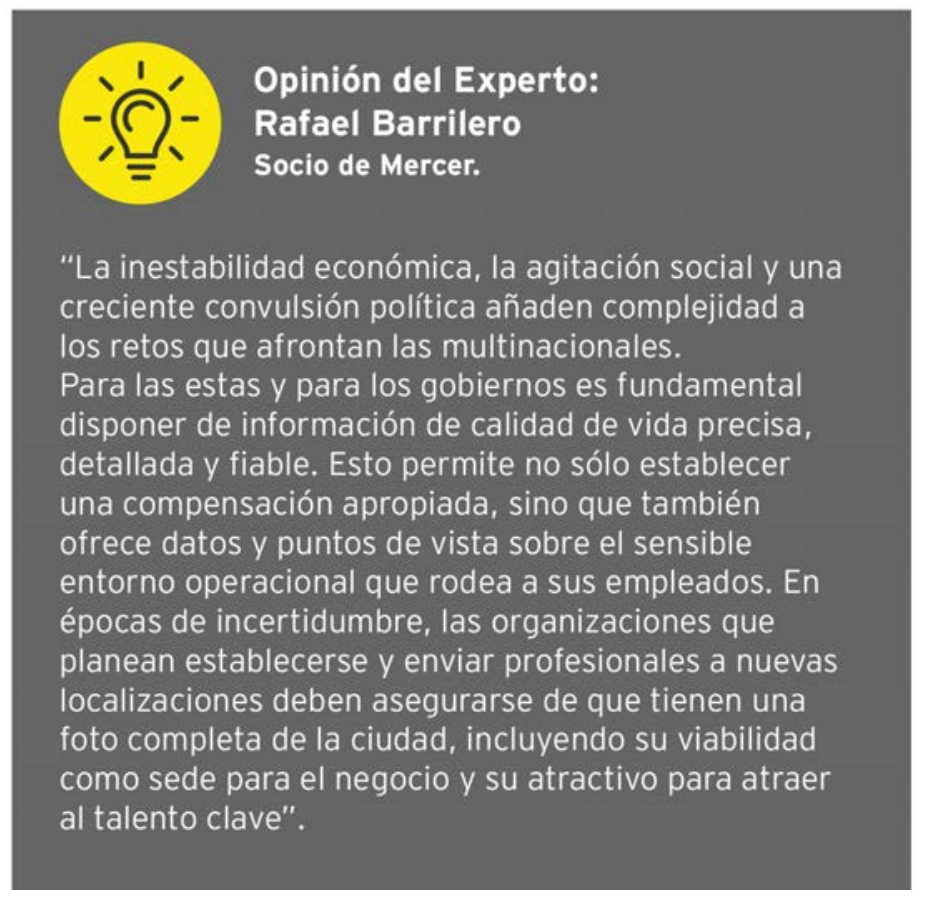




\section{Estado General de las Ciudades}

\section{Comparación de Economías}

En este rubro el referente analizado es el Doing Business, que proporciona una medición objetiva de las normas que regulan la actividad empresarial y su aplicación en 190 economías y sus ciudades en el ámbito subnacional y regional.

\begin{tabular}{|c|l|}
\hline$\#$ & Ciudad \\
\hline 1 & Panamá (Panamá) \\
\hline 2 & San José (Costa Rica) \\
\hline 3 & Guatemala (Guatemala) \\
\hline 4 & Santo Domingo (República Dominicana) \\
\hline 5 & Puerto Cortés (Honduras) \\
\hline 6 & San Pedro Sula (Honduras) \\
\hline 7 & Higüey (República Dominicana) \\
\hline 8 & Soyapango (El Salvador) \\
\hline 9 & San Salvador (El Salvador) \\
\hline 10 & Dajabón (República Dominicana) \\
\hline 11 & Tegucigalpa (Honduras) \\
\hline 12 & Santa Ana (El Salvador) \\
\hline 13 & Quetzaltenango (Guatemala) \\
\hline 14 & San Miguel (El Salvador) \\
\hline 15 & Estelí (Nicaragua) \\
\hline 16 & Santiago de los Caballeros (República \\
\hline 17 & Dominicana) \\
\hline 18 & Cobcuintla (Guatemala) \\
\hline 19 & León (Nicaragua) \\
\hline 20 & Managua (Nicaragua) \\
\hline 21 & Juigalpa (Nicaragua) \\
\hline 22 & Choluteca (Honduras) \\
\hline & \\
\hline
\end{tabular}

En las 22 ciudades analizadas por el Doing Business Centroamérica y República Dominicana 2015, se refleja que hacer negocios es más fácil en Panamá (Panamá), San José (Costa Rica) y Ciudad de Guatemala (Guatemala).

La clasificación general de la facilidad para hacer negocios se basa en el promedio de la distancia a la frontera para los 3 indicadores medidos.

- Apertura de un negocio

- Manejo de permisos de construcción

- Registro de propiedades

En las últimas posiciones encontramos 3 ciudades nicaragüenses y una hondureña.

Los países que conforman la región representan un mercado pequeño, si se compara con México, pero el desarrollo económico de los últimos años y el futuro bono demográfico significan una gran oportunidad comercial.

Finalmente como referencia se incluyen: la mejor y la peor ciudad, desglosadas para cada indicador:

\begin{tabular}{|c|c|c|c|c|}
\hline $\begin{array}{c}\text { Facilidad de hacer } \\
\text { negocios }\end{array}$ & $\begin{array}{c}\text { Apertura de un } \\
\text { negocio }\end{array}$ & $\begin{array}{c}\text { Manejo de permisos de } \\
\text { construcción }\end{array}$ & Registro de propiedades \\
\hline \multirow{1}{*}{ Panamá (Panamá) } & Panamá (Panamá) & León (Nicaragua) & San José (Costa Rica) \\
\hline Choluteca (Honduras) & León (Nicaragua) & Escuintla (Guatemala) & León (Nicaragua) \\
\hline
\end{tabular}




\section{Estado General de las Ciudades}

\section{Infraestructura:}

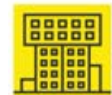

La Comisión Económica para América Latina y el Caribe (CEPAL) indicó que la inversión en infraestructura en América Latina en 2015 alcanzó un 2.2\% del PIB anual en promedio, lo cual es insuficiente para cubrir las necesidades de la región.

De acuerdo a esta deberían invertirse entre 5.0\% y 8.0\% del PIB de América Latina, para lograr superar los rezagos en el desarrollo de infraestructura y convertir a los países integrantes de la región en países competitivos globalmente.

Además es necesario incrementar la inversión para instrumentar políticas públicas integrales, direccionadas a sectores económicamente estratégicos que necesitan ser impulsados para su crecimiento y desarrollo. Los resultados 2017-2018 indican que 12 de los 18 países de la región, se encuentran por debajo de la media mundial con relación a la calidad de su infraestructura.

\begin{tabular}{|c|c|c|c|c|c|c|c|}
\hline País & $\begin{array}{c}\text { Posición } \\
\text { a Nivel } \\
\text { Mundial }\end{array}$ & Carreteras & Ferroviaria & Portuaria & $\begin{array}{c}\text { Aero- } \\
\text { portuaria }\end{array}$ & Eléctrica & $\begin{array}{c}\text { Tele- } \\
\text { comunicaciones }\end{array}$ \\
\hline Panamá & 37 & 49 & 24 & 6 & 11 & 56 & 65 \\
\hline Costa Rica & 65 & 123 & nd & 98 & 64 & 37 & 64 \\
\hline EI Salvador & 77 & 72 & nd & 99 & 99 & 73 & 72 \\
\hline Guatemala & 84 & 106 & nd & 89 & 111 & 42 & 81 \\
\hline Nicaragua & 92 & 54 & nd & 110 & 102 & 87 & 97 \\
\hline Dominicana & 101 & 56 & nd & 38 & 48 & 125 & 75 \\
\hline Honduras & 104 & 81 & nd & 58 & 93 & 104 & 99 \\
\hline
\end{tabular}

A través de una década, poco más del $50 \%$ de los países latinoamericanos han registrado retrocesos en la calidad de su infraestructura.

Los gobiernos deben tener un fuerte compromiso con la transparencia e integridad, de modo que exista un plan para luchar contra la corrupción, de lo contrario no se movilizará la inversión requerida. 


\section{Estado General de las Ciudades}

\section{Población y Salarios}

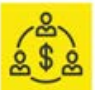

Esta categoría abarca dos temas muy importantes para la región, ambos estrechamente relacionados.

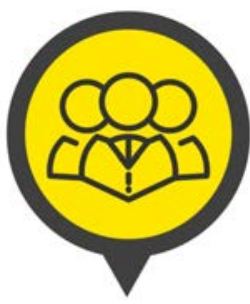

\section{Población}

De acuerdo a un estudio del Banco Mundial las zonas urbanas de Centroamérica viven un acelerado crecimiento que las llevará a concentrar $70 \%$ de la población regional para 2050 , esto representa $\mathbf{3 5}$ millones de personas más que en la actualidad.

Otro informe que muestra resultados similares es el estudio de la Urbanización en Centroamérica: Oportunidades de una Centroamérica Urbana, el cual refleja que la región posee una de las mayores tasas de crecimiento de población urbana, después de África.

Los países que presentan tasas de urbanización anual por encima de la media mundial son Costa Rica con una tasa de $2,5 \%$ y Guatemala con un $3,4 \%$.

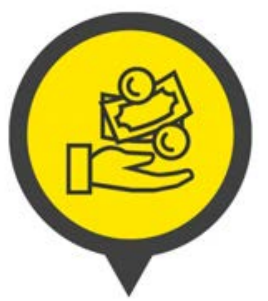

\section{Salarios}

En cada país, la escala del salario mínimo depende del tipo de industria o sector económico. Se ajusta en algunos casos por el tipo de empleado, sus calificaciones o cantidad de empleados de la empresa.

Honduras, Guatemala, El Salvador y Nicaragua tienden a basar su estructura de salarios mínimos por el tipo de industria. Sin embargo, existen diferencias entre los países sobre cual industria paga el salario mínimo más alto.

A nivel regional Costa Rica paga los salarios mínimos más altos y Nicaragua los más bajos, según los ministerios del trabajo de cada país.

Por ejemplo:

En el caso de Costa Rica, el Ministerio del Trabajo y Seguridad Social (MTSS) lo hace según la ocupación específica y el nivel de calificación del trabajador.

Nicaragua, por su parte, divide los salarios mínimos con base al indicador internacional Clasificador Uniforme de Actividad Económica (Cuaen).

\section{Zonas urbanas en Centroamérica concentrarían el} $70 \%$ de la población para el año 2050 


\title{
Estado General de las Ciudades
}

\author{
Centroamerica, Panamá y República Dominicana
}

Finalmente, una de las primeras evaluaciones realizadas a nuestras ciudades es el índice IESE Cities in Motion, creado por la Universidad de Navarra. Para obtener resultados se basa en 69 indicadores, los cuales son capital humano, cohesión social, economía, gestión pública, gobernanza, medio ambiente, movilidad y transporte, planificación urbana, proyección internacional y tecnología. El top 10 de Latinoamérica es el siguiente:

\#3 Ciudad de México: La capital de México se encuentra en la posición

: *87 a nivel mundial. Según el estudio sus políticas medioambientales y su

importancia económica son las principales fortalezas que tiene.

\#8 Monterrey, México: En la posición 111, la capital de Nuevo León aparece entre las 10 mejores por su gobierno y manejo de recursos. medio ambiente le vale a la ciudad de San José . . . : el ubicarse en la posición 112 a nivel mundial.

\#10 Bogotá, Colombia: La capital colombiana ha hecho grandes esfuerzos principalmente en los sectores de gestión pública y planificación urbana. mismos que no pararon desapercibidos para los realizadores del índice.

\#4 Medellín, Colombia: Su desarrollo tecnológico, la movilidad y la planificación urbana le permitieron a Medellín ubicarse como la cuarta mejor en Latinoamérica.

\#2 Santiago, Chile: El estudio resaltó las políticas ambientales y de manejo de recursos tomadas por el gobierno de la capital . . : chilena.

\#6 Sao Paulo, Brasil: La mejor ciudad de Brasil y primera del país en la lista :- tiene como sus principales puntos a favor su proyección internacional y gestión pública.

.

\#5 Montevideo, Uruguay: Montevideo es la última ciudad de la región por .... debajo de la posición 100. Cities in Motion destacó su movilidad y las políticas de medio ambiente.

\#1 Buenos Aires, Argentina: El estudio destacó la eficiencia de su transporte público, así como su gobernanza y proyección internacional.

\#7 Córdoba, Argentina: Destacando por la ... tecnología, Córdoba es la segunda ciudad del país en aparecer en el listado, en la posición 107 a nivel mundial.

Es importante mencionar que las ciudades más inteligentes de América latina empiezan en la posición número 83 del ránking y San José la única representante de Centroamérica.

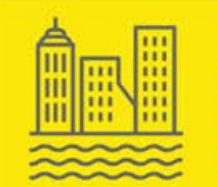

Por su parte EY arrancará con su proceso de Evaluación de las Ciudades del Futuro a nivel regional que comparte algunos indicadores de los mencionados, pero aborda a profundidad los diferentes temas para tener mayores escalas de comprensión. Además permite realizar una comparación mundial ya que el índice en Europa se ha aplicado en más de 116 ciudades. 


\section{De cara al futuro.}

\section{Urbanización}

\section{¿El fenómeno de la urbanización importa para el desarrollo?}

De acuerdo al análisis realizado por el Banco Mundial para Centroamérica la respuesta es afirmativa y la principal razón es que genera oportunidades. Las ciudades permiten una mayor eficiencia en la provisión de servicios, fomentan el intercambio de ideas, tecnología y conocimiento e impulsan una mayor especialización y productividad económica.
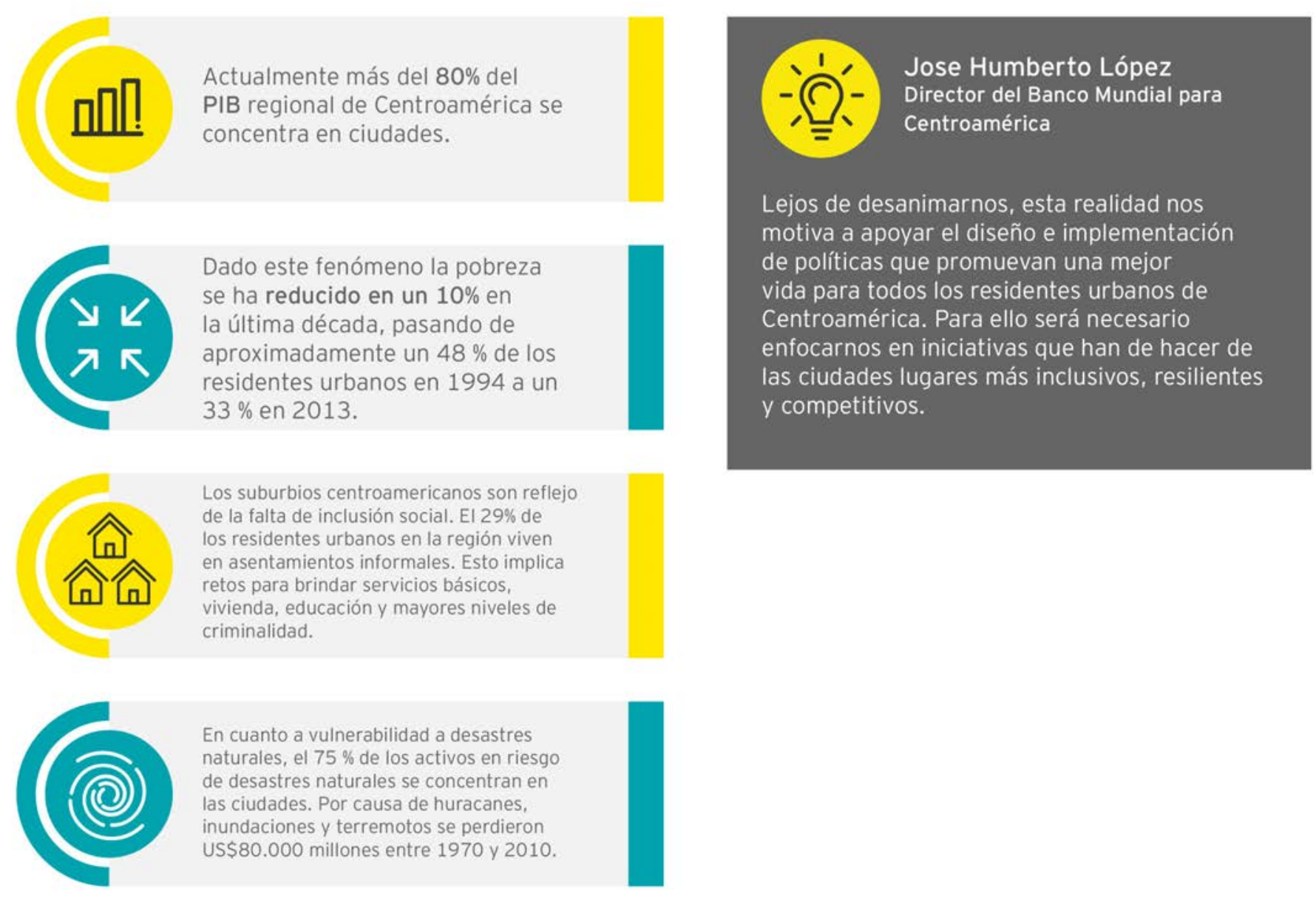

Para que nuestras ciudades sean más inclusivas necesitamos políticas nacionales integrales de vivienda y una mejor articulación con los planes de desarrollo territorial a nivel local.

Para lograr ciudades más resilientes será crucial prevenir pérdidas futuras y tener un mayor entendimiento de los riesgos de desastres naturales para reducir la vulnerabilidad en Centroamérica.

Por último, y para lograr ciudades más competitivas, será fundamental facilitar el acceso de las firmas locales a mecanismos de apoyo a empresas, fomentar el desarrollo de mayores capacidades e innovación y entender mejor las ventajas económicas comparativas, entre otros. 


\section{Retos de las Ciudades}

\section{Centroamerica, Panamá y Republica Dominicana}

Finalmente para complementar el análisis realizado en cada una de las categorías el equipo de EY ha generado un resumen de los retos que enfrentan las ciudades en la región por temas específicos.

\section{Retos de las ciudades}

\begin{tabular}{|c|c|c|c|}
\hline $\begin{array}{l}\text { Transporte } \\
\text { Tra }\end{array}$ & $\begin{array}{l}\text { Seguridad y } \\
\text { protección }\end{array}$ & Servicios públicos & $\begin{array}{l}\text { Educación } \\
\text { Edurion }\end{array}$ \\
\hline $\begin{array}{l}\text { * Productividad perdida } \\
\text { debido a congestión del } \\
\text { tráfico }\end{array}$ & $\begin{array}{l}\text { Crimen, agitación social, } \\
\text { violencia, terrorismo }\end{array}$ & $\begin{array}{l}\text { Aumento de la competencia } \\
\text { por recursos finitos }\end{array}$ & $\begin{array}{l}\text { Encontrar un "modelo de } \\
\text { propósito" del siglo XXI de } \\
\text { la educación }\end{array}$ \\
\hline $\begin{array}{l}\text { * Aumento de la } \\
\text { contaminación en las zonas } \\
\text { urbanas }\end{array}$ & $\begin{array}{l}\text { * Cyber hacking de la } \\
\text { infraestructura crítica }\end{array}$ & $\begin{array}{l}\text { * Ineficiencias en los } \\
\text { sistemas de distribución }\end{array}$ & $\begin{array}{l}\text { * Requerimientos } \\
\text { tecnológicos para la } \\
\text { educación }\end{array}$ \\
\hline $\begin{array}{l}\text { Envejecimiento de los } \\
\text { activos de infraestructura } \\
\text { de transporte }\end{array}$ & $\begin{array}{l}\text { * Cyber hacking a los } \\
\text { ciudadanos }\end{array}$ & $\begin{array}{l}\text { Largos tiempos de } \\
\text { recuperación de desastres } \\
\text { naturales }\end{array}$ & \\
\hline $\begin{array}{l}\text { Accidentes y muertes } \\
\text { relacionadas con el tráfico }\end{array}$ & & $\begin{array}{l}\text { * Métodos ecológicamente } \\
\text { inadecuados para la gestión } \\
\text { de residuos }\end{array}$ & \\
\hline 2 & & & 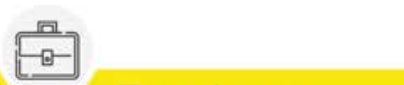 \\
\hline Salud & $\begin{array}{l}\text { Servicios } \\
\text { ciudadanos }\end{array}$ & $\begin{array}{r}\text { Espacios } \\
\text { públicos }\end{array}$ & $\begin{array}{l}\text { Empleo y } \\
\text { desarrollo }\end{array}$ \\
\hline $\begin{array}{l}\text { * Los costos de salud como } \\
\text { porcentaje del PIB son } \\
\text { demasiado altos }\end{array}$ & $\begin{array}{l}\text { * Dificultad para } \\
\text { personalizar los servicios } \\
\text { públicos para el ciudadano }\end{array}$ & $\begin{array}{l}\text { * Información insuficiente } \\
\text { para la planificación urbana }\end{array}$ & $\begin{array}{l}\text { * Ecosistema de startup } \\
\text { limitado y sin énfasis en la } \\
\text { innovación }\end{array}$ \\
\hline $\begin{array}{l}\text { Malos hábitos de vida } \\
\text { y salud que conducen } \\
\text { a problemas de salud } \\
\text { prevenibles }\end{array}$ & $\begin{array}{l}\text { * Demanda -disponibilidad } \\
\text { de los servicios públicos } \\
\text { (gestión de la capacidad) }\end{array}$ & $\begin{array}{l}\text { * Mala gestión de la energía } \\
\text { en los edificios }\end{array}$ & $\begin{array}{l}\text { * Acceso limitado a los } \\
\text { flujos de financiación para } \\
\text { proyectos inteligentes }\end{array}$ \\
\hline $\begin{array}{l}\text { Aumento de la densidad } \\
\text { de población en las zonas } \\
\text { urbanas }\end{array}$ & & $\begin{array}{l}\text { * Disponibilidad limitada de } \\
\text { aparcamiento en las zonas } \\
\text { públicas }\end{array}$ & $\begin{array}{l}\text { * Poco atractivo de } \\
\text { Inversiones Directas } \\
\text { Extranjeras (IED) }\end{array}$ \\
\hline
\end{tabular}

* Temas en los que EY a nivel global ha propuesto soluciones o desarrollado proyectos de Ciudades del Futuro. 


\section{Opinión}

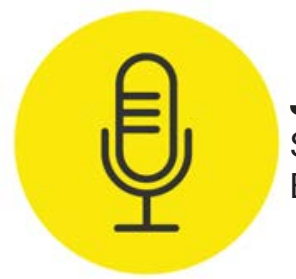

\section{Jennifer Alfaro Portuguez}

Senior Manager Future Cities Team

EY Central America

Los retos, tendencias y contexto actual nos permiten comprender que las ciudades deben priorizar su transformación a ciudades del futuro, de modo que puedan responder en forma oportuna a la era digital y la demanda de sus ciudadanos, el cambio es inevitable y para no quedarse por fuera arrancar ahora es clave. EY les propone a los gobiernos locales identificar su nivel de madurez como ciudad del futuro y con base a ello, alineado a su realidad y necesidades de sus habitantes construir un mapa de ruta que contemple:

Preparación de las infraestructuras básicas, energía y transporte

Profunda comprensión de las circunstancias sociopolíticas

Dimensión comunitaria, necesidades y objetivos de las principales partes interesadas

Reglas, estándares y procesos locales

Modelos de gestión, viabilidad económica y financiera

5. Identificación y aprovechamiento de alianzas estratégicas

\section{¿Qué tipo de ciudad ocupamos?}

Ciudades inteligentes y resilientes, que se definan como:

“Ciudades seguras, donde todas las personas se unen, son amistosas, respetan la infraestructura, detienen la contaminación y cuidan el medio ambiente. Son buenas para tratar con grandes problemas. Son ciudades en paz"

The Rockefeller Foundation 


\section{EY | Assurance | Tax | Transactions | Consulting}

\section{Acerca de EY}

EY es un líder global en servicios de auditoría, impuestos,

transacciones y consultoría. Nuestro conocimiento, puntos de vista y servicios de calidad ayudan a generar confianza en los mercados de capital y en las economías alrededor del mundo. Desarrollamos líderes excepcionales que trabajan en equipo para cumplir las promesas hechas a todos nuestros grupos de interés. Al hacerlo, desempeñamos un papel fundamental en la construcción de un mejor mundo de negocios para nuestra gente, nuestros clientes y nuestras comunidades.

EY se refiere a la organización global de firmas miembro conocidas como Ernst \& Young Global Limited, en la que cada una de ellas actúa como una entidad legal separada. Ernst \& Young Global Limited, compañía del Reino Unido limitada por garantía, no provee servicios a clientes. Más información en www.ey.com.

(c) 2018 E\&Y Central America Inc.

Todos los derechos reservados.

Esta publicación contiene información en forma de resumen y, por lo tanto, su uso es solo para orientación general. No debe considerarse como sustituto de la investigación detallada o del ejercicio de un criterio profesional. Ni E\&Y Central America Inc., ni ningún otro miembro de la organización global de EY acepta responsabilidad alguna por la pérdida ocasionada a cualquier persona que actúe o deje de actuar como resultado de algún contenido en esta publicación. Sobre cualquier asunto en particular, referirse al asesor apropiado.

Los puntos de vista de terceros expuestos en la presente publicación no necesariamente son los puntos de vista de la organización global de EY o de sus firmas integrantes. Por ende, dichos puntos de vista se deben tomar en el contexto del momento en que se expresaron.

ey.com

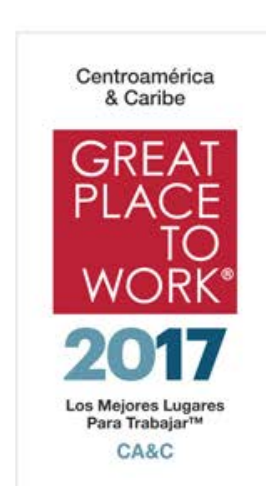

Contactos EY Centroamérica, Panamá y República Dominicana.

\section{Carlos Gallegos}

Leader Others I LATAM North

+ (506) 2208-9800

Carlos.gallegos@cr.ey.com

\section{Laureen Fernández}

Business Development

I Inteligencia de Negocios BD\&C

+ (506) 2208-6693

laureen.fernandez@cr.ey.com

\section{Jennifer Alfaro Portuguez}

Senior Manager

| Future Cities Team

+(506) 2208-9800

Jennifer.Alfaro.Portuguez@cr.ey.com 